\title{
Qué Puede Contribuir el MMPI-2 al Diagnóstico Dual de los Dependientes de Sustancias?
}

What Can Contribute the MMPI-2 of Dual Diagnosis Substance Dependent?

\author{
Pablo González Romero ${ }^{1}$, Guadalupe Sánchez Crespo ${ }^{2}$, Amada Ampudia Rueda ${ }^{3}$ y Fernando \\ Jiménez Gómez ${ }^{4}$
}

\section{Resumen}

El objetivo de este estudio es contrastar las diferencias de trastornos de la personalidad que muestra un grupo Clínico y otro Dependiente de sustancias, mediante el MMPI-2. Con un método descriptivo se analizan los resultados de tres grupos ya establecidos con un mismo número de participantes (113), género y edades: grupo Normal (sin evidencia de patología) grupo Clínico (con trastornos psicológicos que necesitan los servicios de un Centro de Salud) y Dependiente de sustancias (con problemas de adicción a sustancias e internas en una comunidad terapéutica). Se utilizaron los análisis con los estadísticos descriptivos, la $d$ de Cohen y la Curva ROC. Los resultados mostraron la precisión diagnóstica y el poder predictivo de las principales variables del MMPI-2. El MMPI-2 confirma que, entre los grupos Dependiente y Clínico, no existen diferencias significativas en la tétrada psicótica y que sí existen características muy significativas del grupo Dependiente.

Palabras clave: MMPI-2, comorbilidad, cbuso de sustancias, curva ROC

\begin{abstract}
The objective of this study is to compare the differences of personality disorders that shows a group of clinical and other substances, using the MMPI-2 dependent. With a descriptive method are analyzed the results of three groups already set up with the same number of participants (113), gender and age: Normal Group (no evidence of disease) clinical group (with psychological disorders who need the services of a Health Centre) and dependent on substances (with problems of addiction to substances and internal in a therapeutic community). The analyses were used with descriptive statistics, $d$ Cohen and the ROC curve. The results showed the diagnostic accuracy and predictive power of the main variables of the MMPI-2. The MMPI-2 confirms that, between dependent and clinical groups, there are no significant differences in psychotic Tetrad and that there are very significant characteristics of the dependent group.
\end{abstract}

Keywords: MMPI-2, comorbidity, substance abuse, ROC curve

\footnotetext{
1 Doctor en Psicología. Universidad de Salamanca, España. Correo: pgromeiro@gmail.com

2 Facultad de Psicología, Universidad de Salamanca. Avda. de la Merced, 101 37005. Salamanca, España. Correo: lupes@usal.es

3 Facultad de Psicología, Universidad Nacional Autónoma de México (UNAM). Av. Universidad, Av. de los Insurgentes Sur, Copilco Universidad, Coyoacán, 04360 Ciudad de México, D.F. Correo: amada@servidor.unam.mx

${ }^{4}$ Facultad de Psicología, Universidad de Salamanca. Avda. de la Merced, 101 37005, Salamanca, España. Correo: fjimenez@usal.es
} 


\section{Introducción}

Aunque la patología dual, como tal, no es un término reconocido oficialmente por los sistemas de clasificación DSM o CIE, sin embargo los pacientes con dependencia de sustancias se presentan con patología concomitante y en una frecuencia muy superior a la población normal (DeLeon, 1989; Polineni, Moore, \& Guenert, 2010; Verheul, 2001). Los pacientes diagnosticados de patología dual suelen ser diagnosticados, al mismo tiempo, de una enfermedad mental y un trastorno por abuso de sustancias o adicción (Kendall, 2004). El hecho de que la patología dual sea tan frecuente ha suscitado, en nuestro país, el interés y la atención de dos tipos de centros asistenciales fundamentalmente: Centros de Atención al Drogodependiente y Centros de Salud o Servicios de psiquiatría en distintos hospitales y, a veces, con programas de tratamiento e investigaciones organizados de forma independiente (Roberts \& Jones, 2012; Szerman et al., 2011). Además, cada trastorno tiene su propia tasa de prevalencia y comorbilidad (Westermeyer, 2006), y esto puede crear cierto desconcierto, pero aún puede ser más confuso cuando se trata de una patología dual. El estudio piloto llevado a cabo por Szerman et al. (2011) con 400 pacientes en tratamiento procedentes de Centros de Atención al Drogodependiente y de Salud de la comunidad de Madrid (España), pone de manifiesto su preocupación por valorar la presencia de diagnósticos comórbidos de trastornos mentales y adictivos planteando la prevalencia de patología dual en las redes asistenciales entre $34 \%$ y $36.78 \%$ frente a un $28.78 \%$ en la red de Salud mental.

En la labor de la evaluación clínica con los pacientes que abusan de sustancias se debe tener un doble objetivo: en primer lugar, identificar y evaluar el patrón de uso y abuso de sustancias; y en segundo lugar, evaluar los factores de la personalidad y la psicopatología asociada que se sabe están relacionados con el consumo de sustancias. Cuando la tarea de evaluación se concibe de esta manera, es fácil entender cómo el MMPI-2 ofrece muchas ventajas sobre otras medidas de abuso de sustancias. El MMPI-2 ha sido ampliamente utilizado en estudios de pacientes dependientes de sustancias (Egger, Gringhuis, DeMey, Derksen, \& Hilberink, 2007; Forbey \& Ben-Porath, 2007; Gerra, Bertaca, Zaimovic, Pirani, Branchi, \& Ferri, 2008; Rouse, Butcher, \& Miller, 199) con una serie de escalas específicas que evalúa los problemas de abuso de sustancias: la Escala Revisada de Alcoholismo de MacAndrew (Mac-r; Mac Andrew, 1965; 1981), la Escala de Adicción Potencial y la Escalas de Reconocimiento de la Adicción (APS \& AAS; Weed, Butcher, McKenna, \& Ben-Porath, 1992).

Pero sólo la aportación de estas escalas específicas de consumo de sustancias no merecería aplicar el MMPI-2 ya que puede proporcionar también información relevante sobre el nivel emocional del paciente (depresión, autoestima, ansiedad o ira), los desajustes sociales (trastornos de conducta, responsabilidad social o problemas de sociabilidad), apoyos familiares o conyugales, o signos de deterioro cognitivo (esquizofrenia, paranoia, obsesión) que a veces se produce como consecuencia del abuso de sustancias (Ciraulo, Piechniczek- Buczek, \& Iscan, 2003). Estas escalas pueden aportar información relevante para realizar un buen programa terapéutico, último objetivo del internamiento en la comunidad.

En este estudio se ha utilizado la metodología de la curva ROC, para detectar la precisión diagnóstica del MMPI-2 con el conjunto de variables psicológicas integrantes en la prueba. $\mathrm{La}$ utilización, cada vez más frecuente, con el MMPI/MMPI-2 (Burgueño, García-Bastos, \& González-Buitrago, 1995; Glassmire, Kinney, Greene, Stolberg, Berry, \& Cripe, 2003; Jiménez, Sánchez, \& Ampudia, 2008, 2009; Jiménez, Sánchez, \& Tobón, 2009; Nicholson, Mouton, Bagby, \& Buis, 1997; Swets \& Pickett, 1982) se especifica globalmente por el Área Bajo la Curva (AUC) y gráficamente mediante una curva en la que se observan todos los valores de Sensibilidad y Especificidad resultantes de la variación continua de los puntos de corte (Zweig \& Campbell, 1993).

El objetivo de este estudio es contrastar las diferencias de trastornos de la personalidad que muestra un grupo Clínico y otro Dependiente de sustancias, mediante el MMPI-2. 


\section{Método}

\section{Participantes}

El total de participantes fueron 339 con una media de edad de 35.14 años $(S D=8.715)$ y un rango entre 19 y 55 años divididos en tres grupos de 113 (93 varones y 20 mujeres) cada uno:

Grupo Normal. Son 113 personas sin evidencia de patología, 93 varones con una media de edad de 34.00 años $(S D=10.514)$ y un rango establecido entre 19 y 55 años. Las 20 mujeres tienen una media de edad de $36.30(S D=8.898)$ con un rango de edades entre 26 y 51 años.

Grupo Clínico. Son 113 participantes diagnosticados con algún tipo de trastorno psicológico que necesitan la ayuda del profesional (psicólogo/psiquiatra) de un Centro de Salud. Los 93 varones presentan una media de edad de 33.45 años $(S D=8.396)$ y un rango de edad entre 21 y 53 años y las 20 mujeres presentan una media de edad de $37.20 \quad(S D=7.186)$ con edades comprendidas entre 27 y 51 años. Este grupo de pacientes clínicos habían sido diagnosticados previamente por los servicios de su Centro de Salud Mental con diferentes tipos de trastornos: del espectro de la Ansiedad (20\%), del espectro de la Esquizofrenia (16\%), por Dependencia (12\%), Trastornos de Conducta (7\%), de Depresión (12\%) trastorno Somatomorfo (5\%), de la Conducta alimentaria (7\%), Adaptativo (5\%), Emocional (7\%) y otros trastornos (9\%).

Grupo dependiente de sustancias: Son 113 pacientes diagnosticados de un trastorno de dependencia de sustancias e internos en una comunidad terapéutica en proceso de tratamiento. Los 93 varones tienen una media de edad de 37.16 $(S D=7.180)$, con un rango de edad entre 21 y 53 años; las 20 mujeres tienen una edad media de 35.65 años $(S D=7.132)$ con un rango de edades entre 26 y 51 años. La principal sustancia que motiva el tratamiento en los varones es la Heroína (48.39\%), Cocaína (36.71\%) y el Alcohol $(12.90 \%)$, mientras que en las mujeres es la Heroína (55\%), Cocaína (15 \%) y el Alcohol (30\%). El tiempo que llevaban consumiendo los varones: entre 2 y 10 años (17.20\%), entre 11 y 20 años $(46.24 \%)$ y entre 21 y 35 años $(36.56 \%)$. Las mujeres, entre 2 y 10 años (50\%), entre 11 y 20 (40\%) y entre 21 y 35 años (10\%). El $49.56 \%$ había recibido tratamiento psiquiátrico previo a su ingreso en la comunidad terapéutica, mientras que el $50.44 \%$ no lo había recibido. En el momento de ser evaluados, todos los sujetos incluidos en este estudio estaban en tratamiento en la Comunidad Terapéutica "Alborada" de la provincia de Pontevedra, Galicia-España por abuso de sustancias. Los criterios de inclusión para participar en este estudio fueron: a) ser mayor de 18 años y estar en tratamiento por alguna de las sustancias citadas con anterioridad; b) cumplir los criterios de dependencia de sustancias (heroína, cocaína o alcohol) expuestos en el Manual Diagnóstico y Estadístico de los Trastornos Mentales (DSM-IV-R., 2002); c) encontrarse, en el momento de la evaluación, desintoxicados de la droga demandada como tratamiento, es decir, entre dos y cinco semanas después de haber consumido la sustancia por última vez y haber superado el período de abstinencia física. d) participar voluntaria y desinteresadamente.

\section{Procedimiento}

En su diseño, se utilizó el método descriptivo para encontrar las diferencias de puntuaciones medias entre los tres grupos en sus respuestas a los diferentes conjuntos de escalas del MMPI-2. Se añadió el Tamaño del efecto ( $d$ de Cohen, 1988) y la valoración de la precisión diagnóstica, mediante la curva ROC, de las variables MMPI-2 que resultaron ser significativas.

Para homogeneizar las muestras de los diferentes grupos, tanto en número (113), como en género (93 varones y 20 mujeres) y en edades (rango entre 33-37 años) establecida por el grupo de personas dependientes, la asignación a los grupos Normal y Clínico se obtuvieron de forma aleatoria de un conjunto de participantes mucho más amplio que formaba la base de datos en el proceso de la adaptación española del MMPI-2 (Ávila \& Jiménez, 1999). Debido al reducido número de mujeres participantes, se decidió, en los análisis estadísticos de este estudio, tomar el conjunto y no seccionarlos por género.

Teniendo en cuenta la posibilidad de engaño de las personas con problemas de adicción (Kewin \& Nathan, 2006) y garantizar la validez de las respuestas al Cuestionario MMPI-2 se anularon los protocolos cuyas escalas de Validez mostraran valores de ? $=\geq 30 ; \mathrm{L} \geq 80 \mathrm{~T} ; \mathrm{F}$ y $\mathrm{Fb} \geq 100 \mathrm{~T}$ : $\mathrm{K}$ $\geq 70 \mathrm{~T}$; VRIN y TRIN $\geq 80 \mathrm{~T}$ (Butcher, Hess, 
Greene, \& Nelson, 2015). Para garantizar su objetividad, todos los protocolos de respuestas se contestaron en un modelo de hoja mecanizada para su corrección mediante lectora óptica e interpretación a través de un programa informatizado (Leycotest) elaborado expresamente para la interpretación y baremación de los datos extraídos de la lectora. Con ello se analizaron un total 87 variables integradas en los diferentes conjuntos de escalas del MMPI-2

Para poder obtener datos del poder predictivo de la técnica empleada, en este estudio se ha tomado como tasa de prevalencia la referencia dada por Szermann et al. (2011): 30-35\%.

\section{Instrumentos}

El único instrumento administrado a cada una de las personas integrantes en los diferentes grupos, ha sido el Cuestionario de Personalidad de Minnesota -2 (Minnesota Multiphasic Personality Inventory-2), en su adaptación española de Ávila y Jiménez (1999), como medida de autoinforme bien estandarizada y diseñada para evaluar la psicopatología en la personalidad.

\section{Resultados}

Mediante los resultados obtenidos por la diferencia de medias y el tamaño del efecto (Cohen, 1988) se ha tratado de identificar aquellas variables que han resultado ser estadísticamente significativas $(p<.001)$ entre los grupos Clínico y Dependiente. Posteriormente se analizó su precisión diagnóstica mediante la curva ROC.

Al comparar el grupo Clínico con Dependiente se observa (Tabla 1) que ninguna de las variables de las Escalas Clínicas básicas son estadísticamente significativas (ni siquiera las escalas consideradas clásicamente como la "Tétrada Psicótica", Pa, Pt, Sc, y Ma) y solamente la Desviación Psicopática (Pd) ha resultado ser altamente diferente.

En esta Tabla 2 solamente se exponen el grupo de escalas del MMPI-2 (Contenido y Suplementarias) que resultaron ser estadísticamente significativas entre las puntuaciones medias de los grupos Clínico y Dependiente. Tanto la escala de Práctica antisocial (ASP) como Baja autoestima (LSE), presentan un tamaño del efecto "bajo" (Cohen, 1988). Sin embargo, la Escala de Admisión de Adicción (AAS) y la de alcohol de MacAndrew (Mac-r) obtienen grandes diferencias. Igualmente la escala de Responsabilidad Social (Re) resulta ser estadísticamente diferente, pero el grupo Clínico parece ser más responsable socialmente que el grupo Dependiente.

Se obtuvieron las correlaciones bilaterales de las 10 Escalas Clínicas entre los grupos Clínico y Dependiente, obteniéndose de la tétrada psicótica $(\mathrm{Pa}, \mathrm{Pt}, \mathrm{Sc}$ y $\mathrm{Ma})$ un rango de significativas ( $p>0.001)$ y elevadas correlaciones entre valores de $.410(\mathrm{~Pa} / \mathrm{Ma})$ y $.811(\mathrm{Sc} / \mathrm{Pt})$.

En la Tabla 3 se muestran los principales índices de precisión diagnóstica global de las escalas del MMPI-2 que han resultado ser estadísticamente significativas entre los grupos Clínico y Dependiente: Área Bajo la curva (AUC), Sensibilidad, Especificidad y Poder predictivo, tanto positivo como negativo. Para hallar el cálculo del poder predictivo se necesita conocer la tasa de prevalencia y en este estudio se ha tomado como referencia de la señalada por Szerman, et al. (2011), entre el $30 \%$ y 35\% para la patología dual (Tabla 3).

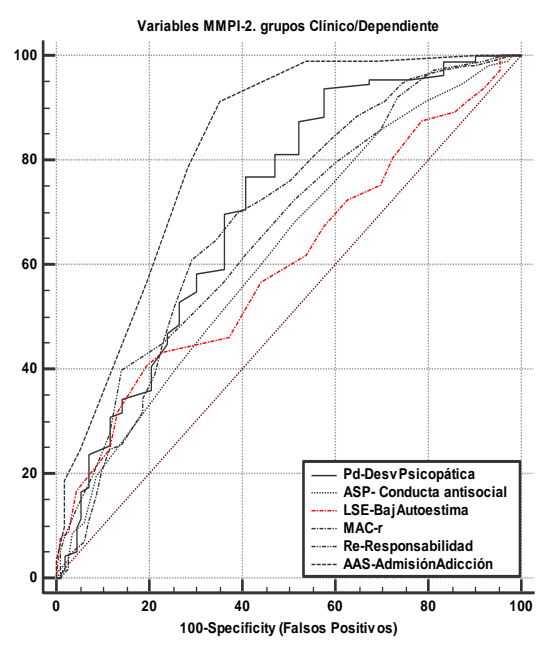

Figura 1. Variables específicas y significativas del grupo Dependiente en su comparación con el grupo Clínico

La Figura 1 muestra la representación gráfica de los valores aportados por la curva ROC de Sensibilidad y Especificidad. Se puede apreciar que la Escala de Adicción de Sustancias (AAS) es la que presenta una mayor precisión global. 
Tabla 1. Escalas Clínicas Básicas (MMPI-2). Diferencias entre grupos de medias y "d" de Cohen

\begin{tabular}{|c|c|c|c|c|c|c|}
\hline Escalas Clínicas básicas & Grupos & Media & D.T. & Comparación grupos & Signif. & $\mathrm{d}$ \\
\hline \multirow{3}{*}{ (Hs) Hipocondría } & Normal & 14.49 & 4.668 & \multirow{3}{*}{$\begin{array}{l}\text { Norm/Depend } \\
\text { Clinico/Depend }\end{array}$} & \multirow{3}{*}{$\begin{array}{l}.005 \\
.126\end{array}$} & \multirow{3}{*}{-0.46} \\
\hline & Clínico & 18.07 & 5.995 & & & \\
\hline & Dependiente & 16.66 & 4.789 & & & \\
\hline \multirow{3}{*}{ (D) Depresión } & Normal & 22.74 & 4.962 & \multirow{3}{*}{$\begin{array}{l}\text { Norm/Depend } \\
\text { Clinico/Depend }\end{array}$} & \multirow{3}{*}{$\begin{array}{l}.000 \\
1.000\end{array}$} & \multirow{3}{*}{$\begin{array}{c}-0.90 \\
-\end{array}$} \\
\hline & Clínico & 27.84 & 6.713 & & & \\
\hline & Dependiente & 27.62 & 5.876 & & & \\
\hline \multirow{3}{*}{ (Hy) Histeria } & Normal & 21.69 & 4.826 & \multirow{3}{*}{$\begin{array}{l}\text { Norm/Depend } \\
\text { Clinico/Depend }\end{array}$} & \multirow{3}{*}{$\begin{array}{l}.000 \\
1.000\end{array}$} & \multirow{3}{*}{$\begin{array}{c}-0.80 \\
-\end{array}$} \\
\hline & Clínico & 25.91 & 5.882 & & & \\
\hline & Dependiente & 25.78 & 5.325 & & & \\
\hline \multirow{3}{*}{ (Pd) Desv.Psicopát. } & Normal & 23.65 & 4.719 & \multirow{3}{*}{$\begin{array}{l}\text { Norm/Depend } \\
\text { Clinico/Depend }\end{array}$} & \multirow{3}{*}{$\begin{array}{l}.000 \\
.000\end{array}$} & \multirow{3}{*}{$\begin{array}{l}-1.90 \\
-0.76\end{array}$} \\
\hline & Clínico & 28.58 & 6.157 & & & \\
\hline & Dependiente & 32.83 & 4.951 & & & \\
\hline \multirow{3}{*}{ (Mf) Mascul/Femen. } & Normal & 26.38 & 5.181 & \multirow{3}{*}{$\begin{array}{l}\text { Norm/Depend } \\
\text { Clinico/Depend }\end{array}$} & \multirow{3}{*}{$\begin{array}{l}.102 \\
1.000\end{array}$} & \multirow{3}{*}{$\begin{array}{r}-0.40 \\
-\end{array}$} \\
\hline & Clínico & 27.85 & 5.202 & & & \\
\hline & Dependiente & 28.44 & 5.177 & & & \\
\hline \multirow{3}{*}{ (Pa) Paranoia } & Normal & 10.52 & 3.538 & \multirow{3}{*}{$\begin{array}{l}\text { Norm/Depend } \\
\text { Clinico/Depend }\end{array}$} & \multirow{3}{*}{$\begin{array}{l}.000 \\
.790\end{array}$} & \multirow{3}{*}{$\begin{array}{c}-1.28 \\
-\end{array}$} \\
\hline & Clínico & 14.68 & 5.294 & & & \\
\hline & Dependiente & 15.33 & 3.979 & & & \\
\hline \multirow{3}{*}{ (Pt) Psicastenia } & Normal & 29.19 & 5.669 & \multirow{3}{*}{$\begin{array}{l}\text { Norm/Depend } \\
\text { Clinico/Depend }\end{array}$} & \multirow{3}{*}{$\begin{array}{l}.000 \\
.090\end{array}$} & \multirow{3}{*}{$\begin{array}{c}-0.81 \\
-\end{array}$} \\
\hline & Clínico & 36.36 & 8.116 & & & \\
\hline & Dependiente & 34.35 & 6.885 & & & \\
\hline \multirow{2}{*}{ (Sc) Esquizofrenia } & Normal & $\begin{array}{l}29.95 \\
4025\end{array}$ & $\begin{array}{l}7.166 \\
11.405\end{array}$ & \multirow{2}{*}{$\begin{array}{l}\text { Norm/Depend } \\
\text { Clinico/Depend }\end{array}$} & \multirow{2}{*}{$\begin{array}{l}.000 \\
.603\end{array}$} & -1.02 \\
\hline & Dependiente & 38.62 & 9.619 & & & \\
\hline & Normal & 18.99 & 4.206 & & & \\
\hline (Ma) Hipomanía & Clínico & 22.09 & 5.380 & Norm/Lepend & .820 & -0.01 \\
\hline & Dependiente & 22.78 & 4.462 & & & \\
\hline & Normal & 28.68 & 8.467 & & & \\
\hline (Si) Introv.Social & Clínico & 32.84 & 9.260 & Clinico/Depend & 959 & \\
\hline & Dependiente & 31.65 & 9.087 & & & \\
\hline
\end{tabular}

Tabla 2. Escalas del MMPI-2 que resultaron ser significativas. Diferencias entre grupos de medias y "d" de Cohen

\begin{tabular}{|c|c|c|c|c|c|c|}
\hline Escalas & Grupos & Media & D.T. & Comparación grupos & Signif. & $\mathrm{d}$ \\
\hline \multirow{3}{*}{$\begin{array}{l}\text { ASP- Conducta } \\
\text { antisocial }\end{array}$} & Normal & 9.92 & 4.097 & \multirow{3}{*}{$\begin{array}{l}\text { Norm/Depend } \\
\text { Clinico/Depend }\end{array}$} & \multirow{3}{*}{. .000} & \multirow{3}{*}{$\begin{array}{l}-0.63 \\
-0.43\end{array}$} \\
\hline & Clínico & 10.76 & 3.776 & & & \\
\hline & Dependiente & 12.35 & 3.578 & & & \\
\hline \multirow{3}{*}{ LSE-BajAutoestima } & Normal & 7.21 & 3.589 & \multirow{3}{*}{$\begin{array}{l}\text { Norm/Depend } \\
\text { Clinico/Depend }\end{array}$} & \multirow{3}{*}{$\begin{array}{l}.010 \\
.010\end{array}$} & \multirow{3}{*}{$\begin{array}{r}-0.41 \\
0.36\end{array}$} \\
\hline & Clínico & 10.91 & 4.944 & & & \\
\hline & Dependiente & 9.06 & 5.352 & & & \\
\hline \multirow{3}{*}{$\begin{array}{l}\text { Mac_r- } \\
\text { AlcoholMacAndrew }\end{array}$} & Normal & 22.25 & 3.826 & \multirow{3}{*}{$\begin{array}{l}\text { Norm/Depend } \\
\text { Clinico/Depend }\end{array}$} & \multirow{3}{*}{$\begin{array}{l}.000 \\
.000\end{array}$} & \multirow{3}{*}{$\begin{array}{l}-1.53 \\
-1.07\end{array}$} \\
\hline & & 23.21 & 5.173 & & & \\
\hline & Dependiente & 28.05 & 3.737 & & & \\
\hline \multirow{2}{*}{$\begin{array}{l}\text { Re-Responsabilidad } \\
\text { Social }\end{array}$} & Normal & 19.72 & 4.348 & \multirow{2}{*}{$\begin{array}{l}\text { Norm/Depend } \\
\text { Clinico/Depend }\end{array}$} & \multirow{2}{*}{$\begin{array}{l}.000 \\
.000\end{array}$} & \multirow{2}{*}{$\begin{array}{l}0.99 \\
0.62\end{array}$} \\
\hline & $\begin{array}{l}\text { Clínico } \\
\text { Dependien }\end{array}$ & 18.14 & $\begin{array}{l}4.480 \\
4364\end{array}$ & & & \\
\hline \multirow{3}{*}{$\begin{array}{l}\text { AAS- } \\
\text { AdmisiónAdicción }\end{array}$} & Normal & 1.75 & 1.634 & \multirow{3}{*}{$\begin{array}{l}\text { Norm/Depend } \\
\text { Clinico/Depend }\end{array}$} & \multirow{3}{*}{$\begin{array}{l}.000 \\
.000\end{array}$} & \multirow{3}{*}{$\begin{array}{l}-2.31 \\
-1.32\end{array}$} \\
\hline & Clínico & 3.19 & 2.407 & & & \\
\hline & Dependiente & 6.23 & 2.196 & & & \\
\hline
\end{tabular}

\section{Discusión y Conclusiones}

El MMPI-2 es una de las pruebas tradicionales de evaluación de la psicopatología de la personalidad que dispone de unas escalas específicas para evaluar la dependencia de sustancias. Pero se ha de tener en cuenta que "al evaluar a un paciente con problemas de abuso de sustancias, mediante el MMPI-2, que su interpretación no debería centrarse sólo en las escalas características del abuso de sustancias" (Kewin \& Nathan, 2006, p. 370). La capacidad del MMPI-2 para identificar los síntomas y tendencia de conductas que forman parte de síndromes 
Tabla 3. Precisión diagnóstica de las variables específicas del MMPI-2 en el grupo Dependiente. Comparación Clínico / Dependiente

\begin{tabular}{lcccccccccc}
\hline \multirow{2}{*}{ Variable } & \multirow{2}{*}{ AUC } & \multirow{2}{*}{ SE } & \multirow{2}{*}{$95 \% \mathrm{CI}$} & \multirow{2}{*}{$\begin{array}{l}\text { P. de } \\
\text { corte }\end{array}$} & Sens. & Espec.- & \multicolumn{4}{c}{ Prevalencia } \\
\cline { 7 - 11 } & & & & & & & & \multicolumn{3}{c}{$35 \%$} \\
\hline Pd & 0.711 & 0,0344 & $0.647-0.769$ & $>26$ & 93.8 & 42.5 & 41.1 & 94.1 & 46.8 & 92.7 \\
ASP & 0.620 & 0,0370 & $0.553-0.684$ & $>10$ & 68.1 & 48.7 & 36.3 & 78.1 & 41.7 & 73.9 \\
LSE & 0.601 & 0,0376 & $0.534-0.665$ & $\leq 6$ & 40.7 & 80.5 & 47.3 & 76.0 & 53.0 & 71.6 \\
Mac-r & 0.684 & 0,0356 & $0.619-0.744$ & $>9$ & 61.1 & 70.8 & 47.3 & 80.9 & 52.3 & 74.3 \\
Re & 0.668 & 0,0356 & $0.602-0.729$ & $\leq 13$ & 39.8 & 85.8 & 54.7 & 76.9 & 60.2 & 72.6 \\
AAS & 0.821 & 0,0279 & $0.765-0.869$ & $>3$ & 91.2 & 64.6 & 52.5 & 94.5 & 58.1 & 93.1 \\
\hline
\end{tabular}

Nota. Pd: Desviación Psicopática; ASP: Prácticas antisociales; LSE: Baja autoestima; Mac-r: Escala revisada de alcohol de MacAndrew; Re: Responsabilidad social; AAS: Escala de Adicción; AUC: Área Bajo la Curva; SE: Desviación estándar; CI: Intervalo de confianza; Sens.: Sensibilidad; Espec.: Especificidad; PP+: Poder Predictivo Positivo; PP-: Poder Predictivo Negativo.

psicopatológicos es, por supuesto, una de sus grandes fortalezas (Kewin \& Nathan, 2006).

De este estudio se han obtenido dos conclusiones relevantes, la primera: se ha confirmado, con el MMPI-2, la evidencia de patología dual en el grupo de personas con dependencia de sustancias (Dependiente). En la mayoría $(85 \%)$ de las variables psicopatológicas del MMPI-2, aquí analizadas, no se han encontrado diferencias estadísticamente significativas entre los grupos Clínico y Dependiente, ni aún en la clásica tétrada psicótica ( $\mathrm{Pa}, \mathrm{Pt}, \mathrm{Sc}$ y Ma). Otros estudios avalan esta misma conclusión al aplicar el MMPI-2 (Craig, 1979a, 1979b, 1982; Shaffer, Nurco, Hanlon, Kinlock, Duszynski, \& Stephenson, 1988; Polimeni et al., 2010). La segunda conclusión se refiere al grupo de variables del MMPI-2 que han resultado ser estadísticamente diferentes y peculiares de la psicopatología del grupo Dependiente. Esta es una de las fortalezas del MMPI-2 porque además de las escalas específicas de consumo de sustancias (AAS, Mac-r y APS) puede detectar variables de desajustes sociales ( $\mathrm{Pd}$, ASP y $\mathrm{Re}$ ), de problemas cognitivos ( $\mathrm{Sc}$, BIZ; Westermeyer, 2006) o de trastornos emocionales (D, DEP, LSE) que pueden ser claves para la programación terapéutica.

Este estudio ha corroborado la consistencia de las investigaciones sobre las escalas específicas de detección de abuso de sustancias (Rouse, Butcher, \& Miller, 1999; Polimeni, Moore, \& Guenert, 2010), pero se destacan especialmente la Escala de Admisión de Adicción (AAS) y la Escala Revisada de Alcoholismo de MacAndrew (Mac- r). La Escala de Adicción de sustancias (AAS) ha sido la que con mayor precisión diagnóstica se ha mostrado por el MMPI-2 al diferenciar a los Dependientes de los Clínicos con un gran tamaño del efecto $(d=1.32)$. Igualmente su precisión diagnóstica global es elevada a través del Área bajo la curva (AUC=.821), su sensibilidad para detectar a los verdaderos Dependientes $(91.2 \%$ y $8.8 \%$ falsos positivos) y una especificidad para detectar a los Clínicos $(64.6 \%$ y $35.4 \%$ de falsos negativos). Las investigaciones actuales sugieren que una alta puntuación en el AAS es el mejor indicador en el MMPI-2 de los problemas de abuso de sustancias (Kewin \& Nathan, 2006). Sin embargo, en este estudio no se ha encontrado diferencias estadísticamente significativas con la escala de Adicción Potencial (APS), probablemente porque se encuentran en proceso terapéutico. Los resultados aportados por la Escala Revisada de Alcoholismo (Mac-r) demuestra un elevado tamaño del efecto $(d=1.07)$, en su diferencia con el grupo Clínicos (Tabla 2). $\mathrm{Su}$ precisión diagnóstica (AUC=.684; sensibilidad $=61.1 \% ; \quad$ especificidad $=70.8 \%) \quad$ se muestra como bastante aceptable y con la posibilidad de detectar a un $38.9 \%$ de falsos positivos y un $29.2 \%$ de falsos negativos.

Una de las escalas que ha detectado con mayor nitidez el grupo Dependiente es la Desviación Psicopática (Pd) con un tamaño del efecto, $d=0.76$ (Tabla 1) que puede ser considerado como "aceptablemente útil" (Swets, 1979, Swets \& Pickett, 1982). Su valor correlacional más elevado lo muestra con la escala Sc $(r=.602)$. También presenta una alta precisión 
diagnóstica ( $\mathrm{AUC}=.711$ ) con una sensibilidad para detectar a los verdaderos Dependientes del $93.8 \%$ (6.2\% de falsos positivos) y una especificidad del $42.5 \%$ para detectar a los que realmente no son Dependientes ( $57.5 \%$ de falsos negativos). La elevación de la escala Pd en el MMPI-2 concuerda con otros estudios llevados a cabo con personas dependientes (DeLeon, 1988, 1989, 1995; Finn, Sharkansky, Brandt, \& Turcotte, 2000; Kewin \& Nathan, 2006). Sin embargo, la escala de Prácticas Antisociales (ASP), aunque se mostraron resultados diferentes del grupo Clínico, sus valores fueron bajos. La escala de responsabilidad social (Re), con sus valores inferiores en el grupo Dependiente, se ha mostrado estadísticamente diferente del grupo Clínico y con un tamaño del efecto $(\mathrm{d}=0.62)$ considerado como aceptable. La precisión diagnóstica que presenta esta escala, a través del MMPI-2, en su $\mathrm{AUC}=0.668$, puede ser considerada como aceptable con una mejor especificidad (85.8\%) que sensibilidad (39.8\%).

Otra escala que ha demostrado ser característica de la personalidad dependiente es la Baja autoestima (LSE). Para Kewin \& Nathan, (2006) esta variable suele estar relacionada con los síntomas depresivos y altamente correlacionada con las variables depresivas (D y DEP) y no es extraño que se encuentre asociada con el mismo tratamiento (Gottheil, Thornton, \& Weinstein, 2002).

No hay dudas de que el presente estudio deja pendientes una serie de limitaciones que pueden ser objeto de futuras investigaciones. Se han encontrado diferencias con otros estudios, posiblemente porque las muestras y el contexto son diferentes. Igualmente existen limitaciones importantes en el riesgo de no detectar a los falsos positivos o negativos cuando hemos establecido una serie de parámetros referentes a la precisión diagnóstica. De todas formas la evaluación de la patología dual debe ser enfocada, con la experiencia del evaluador, a través de un análisis multidimensional y no sólo con unas escalas determinadas por muy buena que sea la prueba. Su utilidad, en última instancia, dependerá principalmente del contexto en el que se aplica y del futuro uso que se haga de las investigaciones.

\section{Referencias}

Asociación Americana de Psiquiatría (APA, 2002). Manual Diagnóstico y Estadístico de los Trastornos Mentales. DSM-IV-R. Masson: Barcelona.

Ávila, E. A. \& Jiménez, F. (1999). Adaptación española del Cuestionario de personalidad MMPI-2. Madrid: TEA ediciones.

Burgueño, M. J., García-Bastos, J. L., \& González-Buitrago, J. M. (1995). La curva ROC en la evaluación de las pruebas diagnósticas. Medicina Clínica, 104, 661-670.

Butcher, J. N. Hess, G. A., Greene, R. L., \& Nelson, L. D.(2015). Using the MMPI-2 in Forensic Assessment. American Psychological Association (APA).

Ciraulo, D. A., Piechniczek-Buczek, J., \& Iscan, E. N. (2003). Outcome predictors in substance use disorders. Psychiatric Clinics of North America, 26, 381-409. http://dx.doi.org/10.10 16/S0193-953X(02)00106-5

Cohen, J. (1988). Statistical Power Analysis for the Behavioral Sciences (2a . ed). LEA. New Jersey

Craig, R. J. (1979a). Personality characteristics of heroin addicts: A review of the empirical literature with critique: Part I. International Journal of the Addictions, 14, 513-532. http://dx.doi.org/10.3109/1082608790905459 9

Craig, R. J. (1979b). Personality characteristics of heroin addicts: A review of the empirical literature with critique: Part II. International Journal of the Addictions, 14, 607-625. http://dx.doi.org/10.3109/1082608790904189 4

Craig, R. J. (1982). Personality characteristics of heroin addicts: Review of empirical research 1976-1979. International Journal of the Addictions, 17, 227-248. http://dx.doi.org/10.3 109/10826088209071008

DeLeon, G. (1988). The therapeutic community and behavioural science. In B. A. Ray (Ed.), Learning factors in substance abuse, NIDA Research Monograph, No. 84 (pp. 74-99), Rockville, MD: NIDA.

DeLeon, G. (1989). Psychopathology and substance abuse. What is being learned from 
research in therapeutic communities. Journal of Psychoactive Drugs, 21, 177-188.

DeLeon, G. (1995). Therapeutic communities for addictions: A theoretical framework. International Journal of the Addictions, 30, 1603-1645. http://dx.doi.org/10.1080/0279107 2.1989.10472158

Egger, J., Gringhuis, M., DeMey, H., Derksen, J., \& Hilberink, S. (2007). MMPI-2 clusters of alcohol dependent patients and the relation to Cloninger's temperament-character inventory. Acta Neuropsychiatrica, 19, 238-243. http://dx.doi.org/10.1111/j.1601-5215.2007.00 188.x

Finn, P. R., Sharkansky, E. J., Brandt, K. M., \& Turcotte, N. (2000). The effects of familial risk, personality, and expectancies on alcohol use and abuse. Journal of Abnormal Psychology, 109, 122-133. http://dx.doi.org/1 0.1037/0021-843X.109.1.122

Forbey, J., \& Ben-Porath, Y. (2007). A comparison of the MMPI-2 restructured clinical (RC) and clinical scales in a substance abuse treatment sample. Psychological Services, 4, 46-58. http://dx.doi.org/10.1037/1 541-1559.4.1.46

Gerra, G., Bertacca, S., Zaimovic, A., Pirani, M., Branchi, B., \& Ferri, M. (2008). Relationship of personality traits and drug of choice by cocaine addicts and heroin addicts. Substance Use \& Misuse, 43, 317-330. http://dx.doi.org/10.1080/1082608070120272 6

Glassmire, D. M., Kinney, D. I., Greene, R. L., Stolberg, R. A., Berry, D. T., \& Cripe, L. (2003). Sensitivity and specificity of MMPI-2 neurologic correction factors: Receiver operating characteristic analysis. Assessment 10, 299-309. http://dx.doi.org/10.1177/107319 1103256129

Gottheil, E., Thornton, C., \& Weinstein, S. (2002). Effectiveness of high versus low structure individual counseling for substance abuse. American Journal on Addiction, 11, 279-290. http://dx.doi.org/10.1080/105504902 90088081

Jiménez, F., Sánchez, G., \& Ampudia, A. (2008). Utilidad de la escala Odecp de Nichols \& Greene (1991) en el MMPI-2. Revista
Iberoamericana de Diagnóstico y Evaluación - e Avaliação Psicológica, 26, 75-91.

Jiménez, F., Sánchez, G., \& Ampudia, A. (2009). La contribución de la Escala PSY-5 al MMPI2. Revista Iberoamericana de Diagnóstico y Evaluación - e Avaliação Psicológica, 2, 3143.

Jiménez, F., Sánchez, G., \& Tobón, C. (2009). A social desirability scale for the MMPI-2. Which of the two: Wiggins (WSD-R) or Edwards (ESD)? The European Journal of Psychology Applied to Legal Context, 1, 147163.

Kendall, C. S. (2004). Treatment of mental illness and comorbid substance abuse: Concepts for evidence-based practice. Journal of Addictions Nursing, 15, 183-186. doi: 10.1080/10884600490888367.

Kevin, R. Y., \& Nathan, C. W. (2006). Assessing alcohol-and drug-abusing clients with the MMPI-2. In James N. Butcher, (Ed). MMPI2: A practitioner's guide, (pp. 361-379). Washington, DC, US: American Psychological Association.

MacAndrew, C. (1965). The differentiation of male alcoholic outpatients from nonalcoholic psychiatric outpatients by means of the MMPI. Quarterly Journal of Studies on Alcohol, 26, 238-246.

MacAndrew, C. (1981). What the MAC scale tells us about men alcoholics: An interpretive review. Journal of Studies on Alcohol, 42, 604-625.

http://dx.doi.org/10.15288/jsa.1981.42.604

Nicholson, R. A., Mouton, G. J., Bagby, M., \& Buis, T. (1997). Utility of MMPI-2 Indicators of response distortion: Receiver operating characteristic analysis. Psychological Assessment, 9, 471-479. http://dx.doi.org/10.1 037/1040-3590.9.4.471

Polimeni, A.-M., Moore, S. M., \& Guenert, S. (2010). MMPI-2 profiles of clients with substance dependencies accessing a therapeutic community treatment facility. Electronic Journal of Applied Psychology. 6, 1-9.

Roberts, B., \& Jones, R. (2012). Dual diagnosis narratives and their implications for the alcohol and other drug sector in Australia. Contemporary Drug Problems 39, 663-685. 
http://dx.doi.org/10.1177/0091450912039004 04

Rouse, S. V., Butcher, J. N., \& Miller, K. B. (1999). Assessment of substance abuse in psychotherapy clients: The effectiveness of the MMPI-2 substance abuse scales. Psychological Assessment, 11, 101-107. http://dx.doi.org/10.1037/1040-3590.11.1.101

Shaffer, J. W., Nurco, D. N., Hanlon, T. E., Kinlock, T., Duszynski, K., \& Stephenson, P. (1988). MMPI-168 profiles of male narcotic addicts by ethnic group and city. Journal of Clinical Psychology, 44, 292-298. http://dx.doi.org/10.1002/10974679(198803)4 4:2\%3C292::AID

Swets, J. A. (1979). ROC analysis applied to the evaluation of medical imaging techniques. Investigative Radiology, 14, 109-121. http://dx.doi.org/10.1097/00004424197903000-00002

Swetts, J. A., \& Pickett, R. M. (1982). Evaluation of diagnostic systems: Methods from signal detection theory. New York: Academic Press.

Szerman B. N., Arias H. F., Vega A. P., Babin V. F., Mesías P. B., Basurte V. I., Morant, C., Ochoa M. E., \& Poyo C. F., (2011). Estudio piloto sobre la prevalencia de patología dual en pacientes en tratamiento en la Comunidad de Madrid. Adicciones, 23, 249-255. http://dx.doi.org/10.20882/adicciones.149

Verheul, R. (2001). Co-morbidity of personality disorders in individuals with substance use disorders. European Psychiatry, 16, 274-282. http://dx.doi.org/10.1016/S09249338(01)00578-8

Weed, N. C., Butcher, J. N., McKenna, T., \& BenPorath, Y. S. (1992). New measures for assessing alcohol and drug abuse with the MMPI-2: The APS and AAS. Journal of Personality Assessment, 58, 389-404. http://dx.doi.org/10.1207/s15327752jpa5802_ 15

Westermeyer, J. (2006). Comorbid schizophrenia and substance abuse: A review of epidemiology and course. American Journal of Addictions, 15, 345-355. http://dx.doi.org/1 0.1080/10550490600860114

Zweig, M. H., \& Campbell, G. (1993). Receiveroperating characteristic (ROC) plots: A fundamental evaluation tool in clinical medicine. Clinical Chemistry, 39, 561-577. 